\title{
Unscented Particle Smoother and Its Application to Transfer Alignment of Airborne Distributed POS
}

\author{
Xiaolin Gong $\mathbb{D},,^{1,2}$ Xiaorui Zheng, ${ }^{1}$ Xing-Gang Yan $\mathbb{D},{ }^{3}$ and Zhaoxing Lu $\mathbb{D}{ }^{1,4}$ \\ ${ }^{1}$ School of Instrumentation Science and Optoelectronics Engineering, Beihang University, Beijing 100191, China \\ ${ }^{2}$ Science and Technology on Inertial Laboratory, Beijing 100191, China \\ ${ }^{3}$ School of Engineering and Digital Arts, University of Kent, Canterbury CT2 7NT, UK \\ ${ }^{4} \mathrm{Xi}$ 'an Institute of Hi-tech, Xi'an 710025, China \\ Correspondence should be addressed to Xiaolin Gong; gongxiaolin@buaa.edu.cn
}

Received 30 January 2018; Accepted 6 August 2018; Published 30 September 2018

Academic Editor: Hikmat Asadov

Copyright (c) 2018 Xiaolin Gong et al. This is an open access article distributed under the Creative Commons Attribution License, which permits unrestricted use, distribution, and reproduction in any medium, provided the original work is properly cited.

\begin{abstract}
This paper deals with the problem of state estimation for the transfer alignment of airborne distributed position and orientation system (distributed POS). For a nonlinear system, especially with large initial attitude errors, the performance of linear estimation methods will degrade. In this paper, a nonlinear smoothing algorithm called the unscented particle smoother (UPS) is proposed and utilized in the off-line transfer alignment of airborne distributed POS. In this algorithm, the measurements are first processed by the forward unscented particle filter (UPF) and then a backward smoother is used to achieve the improved solution. The performance of this algorithm is compared with that of a similar smoother known as the unscented Rauch-Tung-Striebel smoother. The simulation results show that the UPS effectively improves the estimation accuracy and this work offers a new off-line transfer alignment approach of distributed POS for multiantenna synthetic aperture radar and other airborne earth observation tasks.
\end{abstract}

\section{Introduction}

Airborne synthetic aperture radar (SAR) is an important tool for earth observation. Multiantenna SAR can achieve higher accuracy of interferometric processing and ground moving target indication through multibaseline interferometry technology (i.e., interferometric SAR), and it has become an important research direction of radar remote sensing [1]. Usually, these antennae are installed on both sides of the wing of the aircraft, and the motion parameters of each antenna are needed to complete the motion compensation [2]. As a result, airborne distributed position and orientation system (distributed POS) is developed, which is used to provide the time and space reference information for all points of multiantenna SAR or other requirements [3].

Airborne distributed POS is generally composed of four parts: a high-precision POS (main system), a few low-precision inertial measurement units (IMU, subsystem), a POS computer system (PCS), and postprocessing software $[4,5]$. The high precision main system is usually installed in the cabin or underpart of the aircraft and performs the inertial/satellite integrated estimation with the position and velocity of global navigation satellite system (GNSS) as the measurement information. The subsystem, which is placed as close as possible to the measurement center of the load, is used to measure the acceleration and angular velocity which are sent to the PCS. In the PCS, the high precision motion information of main system is used as the reference signal to achieve the motion information of subsystem by transfer alignment technology. The nature of transfer alignment is to estimate and compensate the calculation error of motion parameters of the subsystem by using the high precision information of the main system [6]. It shows that the estimation method is crucial for distributed POS.

It should be noted that distributed POS is a nonlinear system. In some emergency situations, the distributed POS is expected to try to shorten the preparation time on the 
ground and even start up in flight, which will bring the uncertain errors and the misalignment of initial attitude is not small. These make the nonlinearity of the system to be further increased. Thus, the performance of transfer alignment based on the linear model and linear estimation method will degrade [7].

The unscented Kalman filter (UKF) is a typical nonlinear filtering method. It uses a deterministic sampling approach named as sigma points to propagate nonlinear systems and has been discussed in many literatures for the inertial/ satellite integration system [8-10]. UKF is a representative filter to overcome the flaw of truncated error in extended Kalman filter (EKF) [11]. However, the UKF method is an approximation of linear minimum variance estimation and does not apply to general non-Gaussian distributions $[12,13]$. Another popular solution strategy for general filtering problems is particle filter (PF) which is based on the recursive Bayesian estimation and suitable for the nonlinear and non-Gaussian distributed system [14]. The estimated accuracy of PF is higher than the accuracy of using EKF or UKF alone [7]. PF uses a set of sampling points extracted from the posterior probability to express its distribution. Choosing a reasonably recommended probability density is the core of PF. The closer the recommended density selected is to the true density, the better the filter effect is. Otherwise, it is worse or even divergent $[7,15]$. The UKF is able to more accurately propagate the mean and covariance of the Gaussian approximation to the state distribution than the EKF. So PF is combined with UKF and formed a new PF called unscented particle filter (UPF) [15], in which the UKF is used for proposal distribution generation and the performance of the filtering algorithm is improved by taking into account the influence of the measured values on the state estimation.

The above UKF and PF are based on the idea of forward filtering, where only the observation information of the current moment and the previous time are used. The other methods, such as smoothing estimation, can make full use of all the observation information to estimate the state of each moment, and is particularly suitable for offline data processing. Since the smoother uses more observations than the filter, although it cannot be used in real time, the accuracy of the optimal smoothing algorithm is theoretically higher than that of the KF [16]. The unscented Rauch-Tung-Striebel smoother (URTSS) was first proposed by Särkkä in 2008 [17]. This smoother takes the benefit over unscented transformation as well as the smoothing iterative characteristics, which makes the estimation accuracy much better than that of the UKF. In ref. [18], the URTSS is applied to the off-line integrated estimation of the inertial/satellite integration system and shows an obvious accuracy advantage over extended R-T-S smoother in attitude estimation. In particle smoothing, ref. [19] proposed a particle smoothing algorithm which is a direct extension of the PF. This method has the advantage of fixed interval smoothing, but it cannot express the state variables in the past very well. Then, the forward-backward smoother and two-filter smoother based on PF are proposed [20]. This kind of smoothing algorithm needs to carry on the particle filter first and store the particles with weight selected in the approximate process and then perform the smoothing process. However, there is still the problem of particle degeneracy.

For the airborne earth observation system, there are two modes of image processing: real time and off-line. In the off-line cases, there is no high requirement for fast data processing in real-time imaging mode. The smoother can be employed to provide a better solution for off-line image processing. Motivated by these, a smoother called unscented particle smoother (UPS) is proposed in this paper, which is then applied to the off-line transfer alignment of airborne distributed POS. The UPS is made by taking the advantages of the UKF, PF, and smoothing. In the UPS, UKF is used to generate the proposed distributions to overcome the shortage of particle degradation in PF. Then, the forward-backward smoother using all observations is further combined together to obtain higher estimation accuracy. The UPS can be applied to nonlinear and non-Gaussian noise systems as well. There is no need to keep still to obtain the initial motion information of the main system and subsystem before flying off.

The rest of this paper is organized as follows. In Section 2, the UPS are proposed. In Section 3, the design of UPS for airborne distributed POS is given in detail. In Section 4, the performance of the UPS method is demonstrated and compared with that of the URTSS in estimation accuracy by a simulation test. Finally, conclusions are given in Section 5.

\section{Unscented Particle Smoothing Algorithm}

The proposed UPS has two structures: a forward filter and a backward smoother. The forward filter is UPF used to obtain the filtering state estimation. In UPF, the proposed density is determined by UKF, which not only solves the problem of the degradation of particles but also enables particles to get the latest a posteriori information of the measurement vector when they are updated, which is helpful for particles to move toward the area with high likelihood. Then, the backward smoother is conducted after the forward filter to modify the importance weights to achieve the smoothed state estimation.

Suppose the discrete form of the $n_{x}$-state system equation and the observation equation is as follows:

$$
\begin{aligned}
& \mathbf{x}_{k}=f\left(\mathbf{x}_{k-1}, k-1\right)+\Gamma_{k-1} \mathbf{w}_{k-1}, \\
& \mathbf{y}_{k}=h\left(\mathbf{x}_{k}, k\right)+\mathbf{v}_{k},
\end{aligned}
$$

where $\mathbf{x}_{k} \in R^{n_{x}}$ represents the state vector of the system at time $k$ and $\mathbf{y}_{k} \in R^{n_{y}}$ denotes the measurement vector at time $t_{k}$. The functions $f$ and $h$ describe state and measurement models, respectively $[8,21] ; \boldsymbol{\Gamma}_{k-1}$ is the state noise distribution matrix, and $w_{k-1}$ and $v_{k}$ represent the state and measurement noises, respectively, which can be described by the corresponding probability density functions $p\left(w_{k-1}\right)$ 
and $p\left(v_{k}\right)$. In this work, $w_{k-1}$ and $v_{k}$ are assumed to the uncorrelated white Gaussian noise. The mean value of $w_{k-1}$ and $v_{k}$ is zero and their variance matrices are $\mathbf{Q}_{k-1}$ and $\mathbf{R}_{k}$, respectively $[8,22]$.

Based on (1), the process of UPS is described as follows.

Step 1. Initialization $(k=0)[7,15]$. Suppose the initial state variable $x_{0} \sim p\left(x_{0}\right)$, and the covariance matrix is $\mathbf{P}_{0}$.

For $i=1,2, \ldots, N$, draw the states (particles) $x_{0}^{(i)}$ from the prior probability density $p\left(x_{0}\right)$ and set $x_{0}^{(i)} \sim N\left(\widehat{x}_{0}^{(i)}, \mathbf{P}_{0}^{(i)}\right)$, where $\hat{x}_{0}^{(i)}=x_{0}^{(i)}$ and $\mathbf{P}_{0}^{(i)}=\mathbf{P}_{0} . \quad N$ is the number of particles.

Step 2. Importance sampling step (for $i=1,2, \ldots, N$, $k=1,2,3, \ldots, T)$.

(1) Update the particles with the UKF:

(i) Calculate sigma points

$$
\begin{aligned}
& x_{k-1(0)}^{(i)}=\widehat{x}_{k-1}^{(i)}, \\
& \chi_{k-1(j)}^{(i)}=\widehat{x}_{k-1}^{(i)}+\left(\sqrt{\left(n_{x}+\lambda\right) \mathbf{P}_{k-1}^{(i)}}\right)_{(j)} \\
& j=1,2, \ldots, n_{x} \\
& \chi_{k-1(j)}^{(i)}=\hat{x}_{k-1}^{(i)}-\left(\sqrt{\left(n_{x}+\lambda\right) \mathbf{P}_{k-1}^{(i)}}\right)_{(j)} \\
& \quad j=n_{x}+1, n_{x}+2, \ldots, 2 n_{x},
\end{aligned}
$$

where the sigma point set $S=\left\{\chi_{k-1(j)}^{(i)}, W_{j}^{(l)}\right.$; $\left.j=0,1, \ldots, 2 n_{x}, l^{\prime} \in(m, c)\right\}$ is composed of the sigma points $\chi_{k-1(j)}^{(i)}$ and their respective mean $(m)$ and covariance $(c)$ weights $W_{j}^{(l)}$. $\lambda=\alpha^{2}\left(n_{x}+\kappa\right)-n_{x}$ denotes a scaling parameter. The parameter $\alpha$ determines the spread of samples that is usually set between $10^{-4}$ and 1 . The constant $\kappa$ is typically set to 0 or $3-n_{x}$ where $n_{x}$ is the state dimension. The corresponding weights are given by:

$$
\begin{aligned}
& W_{0}^{(m)}=\frac{\lambda}{\left(n_{x}+\lambda\right)}, \\
& W_{0}^{(c)}=\frac{\lambda}{\left(n_{x}+\lambda\right)}+\left(1-\alpha^{2}+\beta\right), \\
& W_{j}^{(m)}=W_{j}^{(c)}=\frac{0.5}{\left(n_{x}+\lambda\right)} \quad j=1,2, \ldots, 2 n_{x},
\end{aligned}
$$

where the parameter $\beta$ is used to incorporate prior knowledge of the distribution (for Gaussian distribution, $\beta=2$ is optimal).

(ii) Time update (propagate particle into future)

$$
\begin{aligned}
\chi_{k \mid k-1(j)}^{(i)} & =f\left(\chi_{k-1(j)}^{(i)}, k-1\right), \\
\hat{x}_{k \mid k-1}^{(i)} & =\sum_{j=0}^{2 n_{x}} W_{j}^{(m)} \chi_{k \mid k-1(j)}^{(i)}, \\
\mathbf{P}_{k \mid k-1}^{(i)}= & \sum_{j=0}^{2 n_{x}} W_{j}^{(c)}\left[\chi_{k \mid k-1(j)}^{(i)}\right. \\
& \cdot\left[\chi_{k \mid k-1(j)}^{(i)}-\widehat{x}_{k \mid k-1}^{(i)}\right] \\
\gamma_{k \mid k-1(j)}^{(i)}= & h\left(\chi_{k \mid k-1(j)}^{(i)}, k\right), \\
\hat{y}_{k \mid k-1}^{(i)}= & \sum_{j=0}^{2 n_{x}} W_{j}^{(m)} \gamma_{k \mid k-1(j)}^{(i)},
\end{aligned}
$$$$
\mathbf{P}_{k \mid k-1}^{(i)}=\sum_{j=0}^{2 n_{x}} W_{j}^{(c)}\left[\chi_{k \mid k-1(j)}^{(i)}-\widehat{x}_{k \mid k-1}^{(i)}\right]
$$$$
\cdot\left[\chi_{k \mid k-1(j)}^{(i)}-\widehat{x}_{k \mid k-1}^{(i)}\right]^{\mathrm{T}}+\boldsymbol{\Gamma}_{k-1} \mathbf{Q}_{k} \boldsymbol{\Gamma}_{k-1}^{\mathrm{T}}
$$

where $\mathbf{P}_{k \mid k-1}^{(i)}$ denotes the prior error covariance.

(iii) Measurement update (incorporate new observation)

$$
\begin{aligned}
\mathbf{P}_{\widehat{y}_{k} \hat{y}_{k}}^{(i)}= & \sum_{j=0}^{2 n_{x}} W_{j}^{(c)}\left[\gamma_{k \mid k-1(j)}^{(i)}-\widehat{y}_{k \mid k-1}^{(i)}\right] \\
& \cdot\left[\boldsymbol{\gamma}_{k \mid k-1(j)}^{(i)}-\widehat{y}_{k \mid k-1}^{(i)}\right]^{\mathrm{T}}+\mathbf{R}_{k},
\end{aligned}
$$

$$
\begin{aligned}
\mathbf{P}_{\widehat{x}_{k} \hat{y}_{k}}^{(i)}= & \sum_{j=0}^{2 n_{x}} W_{j}^{(c)}\left[\chi_{k \mid k-1(j)}^{(i)}-\widehat{x}_{k \mid k-1}^{(i)}\right] \\
& \cdot\left[\gamma_{k \mid k-1(j)}^{(i)}-\widehat{y}_{k \mid k-1}^{(i)}\right]^{\mathrm{T}}, \\
K_{k}^{(i)}= & \mathbf{P}_{\widehat{x}_{k} \hat{y}_{k}}^{(i)} \mathbf{P}_{\widehat{y}_{k} \hat{y}_{k}}^{(i)}-1, \\
\widehat{x}_{k}^{(i)}= & \widehat{x}_{k \mid k-1}^{(i)}+K_{k}^{(i)}\left(y_{k}-\widehat{y}_{k \mid k-1}^{(i)}\right), \\
\mathbf{P}_{k}^{(i)}= & \mathbf{P}_{k \mid k-1}^{(i)}-K_{k}^{(i)} \mathbf{P}_{\hat{y}_{k} \hat{y}_{k}}\left(K_{k}^{(i)}\right)^{\mathrm{T}},
\end{aligned}
$$

where $\widehat{y}_{k \mid k-1}^{(i)}$ denotes the predicted measurement, $\mathbf{P}_{\widehat{y}_{k} \hat{y}_{k}}^{(i)}$ denotes the covariance of predicted measurement error, and $\mathbf{P}_{\hat{x}_{k} \hat{y}_{k}}^{(i)}$ denotes the crosscovariance matrix between $\hat{x}_{k \mid k-1}^{(i)}$ and $\hat{y}_{k \mid k-1}^{(i)}$. $K_{k}^{(i)}$ denotes the filter gain. 


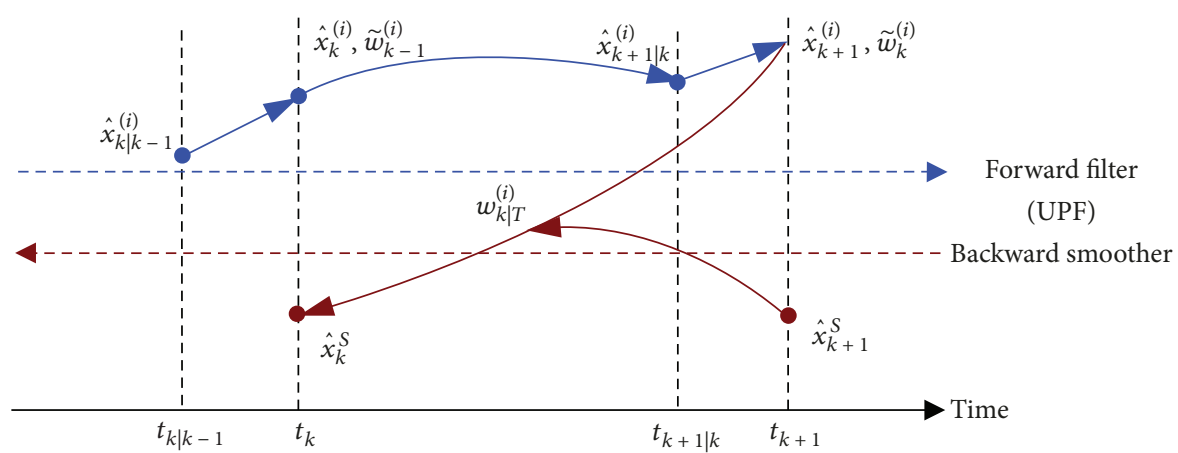

Figure 1: The principle diagram of UPS.

(2) Importance sampling step.

For $i=1,2, \ldots, N$, sample $\tilde{x}_{k}^{(i)} \sim q\left(x_{k}^{(i)} \mid x_{0: k-1}^{(i)}, y_{1: k}\right)=$ $N\left(\widehat{x}_{k}^{(i)}, \mathbf{P}_{k}^{(i)}\right)$ and set $\tilde{x}_{k}^{(i)} \triangleq\left(x_{0: k-1}^{(i)}, \tilde{x}_{k}^{(i)}\right)$ and $\tilde{\mathbf{P}}_{0: k}^{(i)} \triangleq$ $\left(\mathbf{P}_{0: k-1}^{(i)}, \tilde{\mathbf{P}}_{k}^{(i)}\right)$.

(3) Update and normalize the importance weights.

For $i=1,2, \ldots, N$, evaluate the importance weights up to a normalizing constant:

$$
\tilde{w}_{k}^{(i)}=w_{k-1}^{(i)} \frac{p\left(y_{k} \mid \tilde{x}_{k}^{(i)}\right) p\left(\tilde{x}_{k}^{(i)} \mid x_{k-1}^{(i)}\right)}{q\left(\tilde{x}_{k}^{(i)} \mid x_{0: k-1}^{(i)}, y_{1: k}\right)} .
$$

For $i=1,2, \ldots, N$, normalize and store the importance weights:

$$
\tilde{w}_{k}^{(i)}=w_{k}^{(i)}\left[\sum_{i=1}^{N} w_{k}^{(i)}\right]^{-1}
$$

Compute the filtering state estimation $\widehat{x}_{k}^{F}$ :

$$
\widehat{x}_{k}^{F}=\sum_{i=1}^{N} \tilde{w}_{k}^{(i)} \widehat{x}_{k}^{(i)}
$$

Step 3. Using the importance weights stored in the forward filtering to conduct the backward smoothing recursion.

Set $w_{T \mid T}^{(i)}=\bar{w}_{T \mid T}^{(i)}$. For $k=T-1, \ldots, 1$ and $i=1,2, \ldots, N$, evaluate

$$
w_{k \mid T}^{(i)}=w_{k+1 \mid T}^{(i)}\left[\sum_{j=1}^{N} w_{k+1 \mid T}^{(j)} \frac{p\left(\tilde{x}_{k+1}^{(j)} \mid \tilde{x}_{k}^{(i)}\right)}{\sum_{m=1}^{N} \tilde{w}_{k}^{(m)} p\left(\tilde{x}_{k+1}^{(j)} \mid \tilde{x}_{k}^{(m)}\right)}\right] .
$$

(1) Resampling

For $\tilde{x}_{k}^{(i)}(i=1,2, \ldots, N)$, Using sequential importance resampling (SIR) [15] to complete resampling to obtain $N$ random samples $\mathbf{x}_{0: k}^{\prime(i)}$, and set $\mathbf{w}_{k}^{(i)}=\tilde{\mathbf{w}}_{k}^{(i)}=1 / N$.

(2) Compute the smoothed state estimation $\widehat{x}_{k}^{S}$

$$
\widehat{x}_{k}^{S}=\frac{1}{N} \sum_{i=1}^{N} x_{k}^{\prime(i)}
$$

The principle diagram of UPS is shown in Figure 1. In summary, there are two processes: a forward filtering process and a backward smoothing process. The measurements are first processed by the forward filter and the importance weight $\tilde{w}_{k}^{(i)}$ should be stored in the forward filter stage. And then, a separate backward smoothing pass is used to modify the importance weights for obtaining the smoothing solution.

\section{UPS Design for Airborne Distributed POS}

The design of UPS for airborne distributed POS is given in this section. Firstly, the definition of coordinate frames is introduced. $i$ and $e$ denote the earth-center inertial frame and the earth-centered earth-fixed frame, respectively. The navigation frames of the main system and subsystem are both topocentric frames (the $z$-axis parallel to the upward vertical, the $x$-axis pointing eastward, and the $y$-axis pointing northward), represented by $n$ and $n_{s}$, respectively. The body frames of the main system and subsystem are denoted by $b$ and $b_{s}$. A detailed description of these coordinate frames is available in $[18,23]$.

Figure 2 show the block diagram of transfer alignment in distributed POS based on UPS.

In Figure 2, the strapdown navigator calculates the attitude (heading, pitch, and roll), velocity, and position expressed in $n_{s}$-frame by using the angular rates and 


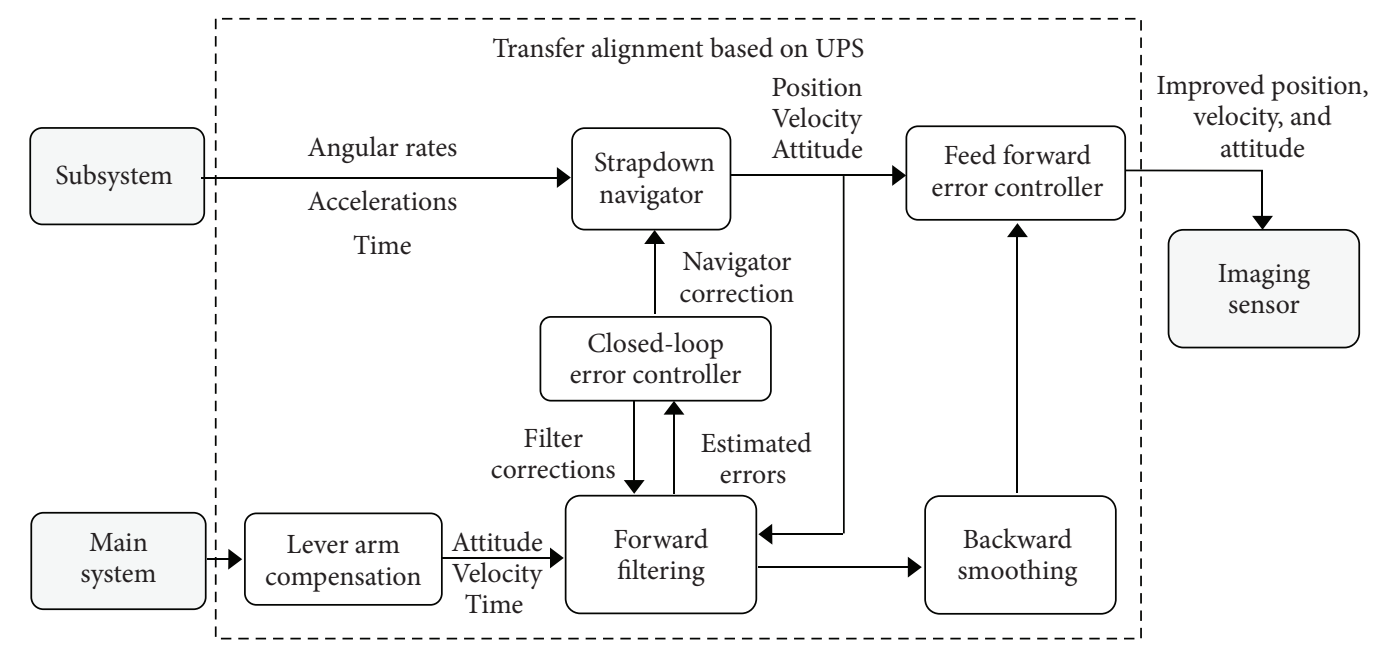

FIGURE 2: The block diagram of transfer alignment in distributed POS based on UPS.

accelerations of subsystem. Since the strapdown navigation is an integration process, any errors of the accelerometers and gyroscopes will integrate into slowly growing errors on velocity, position, and attitude. The differences of velocity and attitude between the main system and the strapdown navigator are used in the forward filtering to estimate the growing velocity, position, and attitude errors in the strapdown navigator. The closed-loop error controller is used to correct the result of strapdown navigator by using the estimation of forward filtering. After the end of the forward filter, the backward smoother is performed to compensate the estimation of forward filtering, and the improved estimation results are used to correct the result of strapdown navigator in the feed forward error controller. The improved attitude, velocity, and position are outputted to the imaging sensor at last.

3.1. Nonlinear Inertial Navigation Error Model. The inertial navigation error equations are the basis of the mathematical model for transfer alignment. According to the definitions above, the nonlinear SINS error model of the subsystem based on angle error, which includes attitude error equation, velocity error equation, position error equation, and inertial senor constant error equation, is given by [24]:

$$
\begin{aligned}
\dot{\phi}^{n_{s}}= & \left(\mathbf{I}-\mathbf{C}_{n_{s}}^{p}\right) \boldsymbol{\omega}_{i n_{s}}^{n_{s}}+\delta \boldsymbol{\omega}_{i n_{s}}^{n_{s}}-\mathbf{C}_{b_{s}}^{n_{s}} \mathbf{\varepsilon}^{b_{s}}, \\
\delta \dot{\mathbf{V}}^{n_{s}}= & \left(\mathbf{I}-\mathbf{C}_{p}^{n_{s}}\right) f^{n_{s}}-\left(2 \delta \omega_{i e}^{n_{s}}+\delta \omega_{e n_{s}}^{n_{s}}\right) \\
& \times \mathbf{V}^{n_{s}}-\left(2 \delta \omega_{i e}^{n_{s}}+\delta \omega_{e n_{s}}^{n_{s_{s}}}\right) \times \delta \mathbf{V}^{n_{s}}+\widehat{\mathbf{C}}_{b}^{n_{s}} \nabla^{b_{s}}, \\
\delta \dot{L}= & \frac{\delta V_{\mathrm{N}}}{R_{\mathrm{M}}+H}-\frac{V_{\mathrm{N}}}{\left(R_{\mathrm{M}}+H\right)^{2}} \delta H, \\
\delta \dot{\lambda}= & \frac{\sec L}{R_{\mathrm{N}}+H} \delta V_{\mathrm{E}}+\frac{V_{\mathrm{E}} \sec L \tan L}{R_{\mathrm{N}}+H} \delta L-\frac{V_{\mathrm{E}} \sec L}{\left(R_{\mathrm{N}}+H\right)^{2}} \delta H,
\end{aligned}
$$

$$
\begin{aligned}
\delta \dot{H} & =\delta V_{U}, \\
\dot{\varepsilon}_{c}^{b_{s}} & =0, \\
\dot{\nabla}_{c}^{b_{s}} & =0,
\end{aligned}
$$

where $\phi^{n_{s}}=\left[\begin{array}{lll}\phi_{\mathrm{E}} & \phi_{\mathrm{N}} & \phi_{\mathrm{U}}\end{array}\right]^{\mathrm{T}}$ denotes the attitude error vector in $n_{s}$-frame, the subscripts $\mathrm{E}, \mathrm{N}$, and $\mathrm{U}$ denote the east, north, and up components, respectively; $\omega_{i n_{s}}^{n_{s}}$ denotes the rotation velocity of the $n_{s}$-frame relative to the $i$-frame expressed in $n_{s}$-frame with error $\delta \omega_{i n_{s}}^{n_{s}} ; \mathbf{C}_{b_{s}}^{n_{s}}$ denotes the coordinate transformation matrix from the $b_{s}$-frame to the $n_{s}$-frame; $\varepsilon^{b_{s}}$ denotes the gyro drift of subsystem in $b_{s}$-frame, which consists of random constant drift $\varepsilon_{c}^{b_{s}}$ and the Gaussian white noise $\omega_{\varepsilon}^{b_{s}}$ with $\varepsilon_{c}^{b_{s}}=\left[\begin{array}{llll}\varepsilon_{x}^{b_{s}} & \varepsilon_{y}^{b_{s}} & \varepsilon_{z}^{b_{s}}\end{array}\right]^{\mathrm{T}}$ and $\omega_{\varepsilon}^{b_{s}}=\left[\begin{array}{lll}\omega_{\varepsilon_{x}}^{b_{s}} \omega_{\varepsilon_{y}}^{b_{s}} \omega_{\varepsilon_{z}}^{b_{s}}\end{array}\right]^{\mathrm{T}} ; \quad \mathbf{V}^{n_{s}}=\left[\begin{array}{lll}V_{\mathrm{E}} & V_{\mathrm{N}} & V_{\mathrm{U}}\end{array}\right]^{\mathrm{T}}$ denotes the velocity vector in $n_{s}$-frame with error $\delta \mathbf{V}^{n_{s}}=$ $\left[\begin{array}{lll}\delta V_{\mathrm{E}} & \delta V_{\mathrm{N}} & \delta V_{\mathrm{U}}\end{array}\right]^{\mathrm{T}} ; f^{n_{s}}=\left[\begin{array}{lll}f_{\mathrm{E}} & f_{\mathrm{N}} & f_{\mathrm{U}}\end{array}\right]^{\mathrm{T}}$ denotes the specific force measured by the accelerometers of subsystem expressed in $n_{s}$-frame; $\omega_{i e}^{n_{s}}$ denotes the rotation velocity of the $e$-frame relative to the $i$-frame expressed in $n_{s}$-frame with error $\delta \omega_{i e}^{n_{s}} ; \omega_{e n_{s}}^{n_{s}}$ denotes the rotation velocity of the $n_{s}$-frame relative to the $e$-frame expressed in $n_{s}$-frame with error $\delta \omega_{e n_{s}}^{n_{s}}$; $\nabla^{b_{s}}$ denotes the accelerometer bias of subsystem in $b_{s}$-frame, which consists of random constant bias $\nabla_{c}^{b_{s}}$ and the Gaussian white noise $\omega_{\nabla}^{b_{s}}$ with $\nabla_{c}^{b_{s}}=\left[\nabla_{x}^{b_{s}} \nabla_{y}^{b_{s}} \nabla_{z}^{b_{s}}\right]^{\mathrm{T}}$ and $\omega_{\nabla}^{b_{s}}=$ $\left[\omega_{\nabla_{x}}^{b_{s}} \omega_{\nabla_{y}}^{b_{s}} \omega_{\nabla_{z}}^{b_{s}}\right]^{\mathrm{T}}$; $\sec L=1 / \cos L$. The symbols $\delta L, \delta \lambda$, and $\delta H$ denote the latitude error, longitude error, and height error, respectively; and $R_{\mathrm{M}}$ and $R_{\mathrm{N}}$ denote the meridian and transverse radius of curvature, respectively.

The nonlinear terms of attitude and velocity error equations are $\left(\mathbf{I}-\mathbf{C}_{n_{s}}^{p}\right) \omega_{i n_{s}}^{n_{s}}$ and $\left(\mathbf{I}-\mathbf{C}_{p}^{n_{s}}\right) f^{n_{s}}$ in (11), respectively, and the nonlinear matrix $\mathbf{C}_{n_{s}}^{p}=\left(\mathbf{C}_{p}^{n_{s}}\right)^{\mathrm{T}}$ is expressed as follows: 


$$
\mathbf{C}_{n_{s}}^{p}=\left[\begin{array}{ccc}
\cos \phi_{\mathrm{N}} \cos \phi_{U}-\sin \phi_{\mathrm{E}} \sin \phi_{\mathrm{N}} \sin \phi_{\mathrm{U}} & \cos \phi_{\mathrm{N}} \sin \phi_{\mathrm{U}}+\sin \phi_{\mathrm{E}} \sin \phi_{\mathrm{N}} \cos \phi_{\mathrm{U}} & -\cos \phi_{\mathrm{E}} \sin \phi_{\mathrm{N}} \\
-\cos \phi_{\mathrm{E}} \sin \phi_{\mathrm{U}} & \cos \phi_{\mathrm{E}} \cos \phi_{\mathrm{U}} & \sin \phi_{\mathrm{E}} \\
\sin \phi_{\mathrm{N}} \cos \phi_{\mathrm{U}}+\sin \phi_{\mathrm{E}} \cos \phi_{\mathrm{N}} \sin \phi_{\mathrm{U}} & \sin \phi_{\mathrm{N}} \sin \phi_{\mathrm{U}}-\sin \phi_{\mathrm{E}} \cos \phi_{\mathrm{N}} \cos \phi_{\mathrm{U}} & \cos \phi_{\mathrm{E}} \cos \phi_{\mathrm{N}}
\end{array}\right]
$$

3.2. Model of Rigid Misalignment Angle and Flexure Angle. During the transfer alignment, there are flexure angles and rigid misalignment angles between the main system and the subsystem. These angles cannot be measured accurately and the flexure angles vary with the time. Therefore, the flexure angles and rigid misalignment angles should be modeled and estimated.

The models of rigid misalignment angles and flexure angles are shown in the following equations:

$$
\begin{aligned}
\dot{\rho}_{j} & =0, \\
\ddot{\theta}_{j}+2 \beta_{j} \dot{\theta}_{j}+\beta_{j}^{2} \theta_{j} & =\eta_{j} \quad j=x, y, z,
\end{aligned}
$$

where $\rho_{j}$ denotes the rigid misalignment angle of subsystem; $\theta_{j}$ denotes the flexure angle and described by the secondorder Markov process [25]; $\beta_{j}=2.146 / \tau_{j}$ and $\tau_{j}$ is the correlation time; $x, y$, and $z$ are the axes of $b_{s}$-frame; $\eta_{j}$ denotes the Gaussian white noise with covariance $Q_{\eta_{j}}=4 \beta_{j}^{3} \sigma_{j}^{2}$; and $\sigma_{j}^{2}$ denotes the covariance of $\theta_{j} ; Q_{\eta}=\left[Q_{\eta_{x}} Q_{\eta_{y}} Q_{\eta_{z}}\right]^{\mathrm{T}}$.

\subsection{Design of the Smoother for Transfer Alignment of Distributed POS}

3.3.1. State Equation. On the basis of models established in Subsections 3.1 and 3.2, let

$$
\begin{aligned}
& x=\left[\begin{array}{ll}
x_{1} & x_{2}
\end{array}\right]^{\mathrm{T}}, \\
& x_{1}=\left[\begin{array}{lllllllllllllll}
\phi_{\mathrm{E}} & \phi_{\mathrm{N}} & \phi_{\mathrm{U}} & \delta V_{\mathrm{E}} & \delta V_{\mathrm{N}} & \delta V_{\mathrm{U}} & \delta L & \delta \lambda & \delta H & \varepsilon_{x}^{b_{s}} & \varepsilon_{y}^{b_{s}} & \varepsilon_{z}^{b_{s}} & \nabla_{x}^{b_{s}} & \nabla_{y}^{b_{s}} & \nabla_{z}^{b_{s}}
\end{array}\right]^{\mathrm{T}}, \\
& x_{2}=\left[\begin{array}{lllllllll}
\rho_{x} & \rho_{y} & \rho_{z} & \theta_{x} & \theta_{y} & \theta_{z} & \dot{\theta}_{x} & \dot{\theta}_{y} & \dot{\theta}_{z}
\end{array}\right]^{\mathrm{T}} \text {. }
\end{aligned}
$$

Then, the continuous-time system model can be given by

$$
\dot{x}=f(x, \mathrm{t})+G(\mathrm{t}) \mathbf{W}_{I}(\mathrm{t}),
$$

where the nonlinear function $f(x, \mathrm{t})$ is determined by error model of transfer alignment in Subsections 3.1 and 3.2, the vector $\mathbf{W}_{I}(\mathrm{t})$ represents the system noise. $\mathbf{W}_{1}=\left[\begin{array}{lllllllll}\omega_{\varepsilon_{x}}^{b_{s}} & \omega_{\varepsilon_{y}}^{b_{s}} & \omega_{\varepsilon_{z}}^{b_{s}} & \omega_{\nabla_{x}}^{b_{s}} & \omega_{\nabla_{y}}^{b_{s}} & \omega_{\nabla_{z}}^{b_{s}} & \eta_{x} & \eta_{y} & \eta_{z}\end{array}\right]^{\mathrm{T}}$ is the zero-mean Gaussian white noise vector with covariance $Q_{I}$ which consists of covariance $Q_{\varepsilon}$ of gyro random drift, covariance $Q_{\nabla}$, of accelerometer random bias, and $Q_{\eta}$.

3.3.2. Measurement Equation. The differences of velocity and attitude between the main system and the subsystem solutions are considered as the measurement vector. The measurement vector can be given as

$$
\mathbf{y}(t)=\left[\begin{array}{llllll}
\delta \psi & \delta \theta & \delta \gamma & \delta V_{\mathrm{E}}^{\prime} & \delta V_{\mathrm{N}}^{\prime} & \delta V_{\mathrm{U}}^{\prime}
\end{array}\right]^{\mathrm{T}},
$$

where $\delta \psi, \delta \theta$, and $\delta \gamma$ denote the differences of heading angle, pitch angle, and roll angle between the main system and the subsystem; $\delta V_{\mathrm{E}}^{\prime}, \delta V_{\mathrm{N}}^{\prime}$, and $\delta V_{\mathrm{U}}^{\prime}$ denote the differences of the east, north, and up velocities between the main system and the subsystem accounting for the lever arm between the main system and subsystem [25].

The measurement vector can be expressed linearly by the state vector shown in (14). Hence, the linear measurement model can be given by

$$
\mathbf{y}(t)=\mathbf{H x}(t)+\mathbf{v}(t)
$$

where the measurement noise $\mathbf{v}(t)=\left[\begin{array}{llll}v_{\delta \psi} & v_{\delta \theta} & v_{\delta \gamma} & v_{\delta V_{\mathrm{E}}^{\prime}}\end{array}\right.$ $\left.v_{\delta V_{\mathrm{N}}^{\prime}} v_{\delta V_{\mathrm{U}}^{\prime}}^{\prime}\right]^{\mathrm{T}}$ is the white noise vector of the solution of the main system with zero mean and covariance matrix $\mathbf{R}$. The measurement matrix $\mathbf{H}(t)$ can be given as

$$
\mathbf{H}(t)=\left[\begin{array}{cccccc}
\mathbf{H}_{1} & 0_{3 \times 3} & 0_{3 \times 9} & \mathbf{H}_{2} & \mathbf{H}_{3} & 0_{3 \times 3} \\
0_{3 \times 3} & \mathbf{I}_{3 \times 3} & 0_{3 \times 9} & 0_{3 \times 3} & 0_{3 \times 3} & 0_{3 \times 3}
\end{array}\right]_{6 \times 24} .
$$


Here, the attitude matrix of main system $\mathbf{C}_{b}^{n}$ is denoted as $\mathbf{T}_{a} . \mathbf{T}_{a}, \mathbf{H}_{1}, \mathbf{H}_{2}$, and $\mathbf{H}_{3}$ are as follows:

$$
\mathbf{C}_{b}^{n}=\left[\begin{array}{ccc}
\cos \gamma_{m} \cos \varphi_{m}-\sin \gamma_{m} \sin \theta_{m} \sin \varphi_{m} & -\cos \theta_{m} \sin \varphi_{m} & \sin \gamma_{m} \cos \varphi_{m}+\cos \gamma_{m} \sin \theta_{m} \sin \varphi_{m} \\
-\cos \gamma_{m} \sin \varphi_{m}+\sin \gamma_{m} \sin \theta_{m} \cos \varphi_{m} & \cos \theta_{m} \cos \varphi_{m} & \sin \gamma_{m} \sin \varphi_{m}-\cos \gamma_{m} \sin \theta_{m} \cos \varphi_{m} \\
-\sin \gamma_{m} \cos \theta_{m} & \sin \theta_{m} & \cos \gamma_{m} \cos \theta_{m}
\end{array}\right]
$$

where $\varphi_{m}, \theta_{m}$, and $\gamma_{m}$ denote the heading, pitch, and roll of the main system, respectively.

$$
\begin{aligned}
& \mathbf{H}_{1}=\left[\begin{array}{ccc}
\frac{T_{\mathrm{a}}{ }^{(12)} T_{\mathrm{a}}{ }^{(32)}}{\left(T_{\mathrm{a}}{ }^{(12)}\right)^{2}+\left(T_{\mathrm{a}}{ }^{(22)}\right)^{2}} & \frac{T_{\mathrm{a}}{ }^{(22)} T_{\mathrm{a}}{ }^{(32)}}{\left(T_{\mathrm{a}}{ }^{(12)}\right)^{2}+\left(T_{\mathrm{a}}{ }^{(22)}\right)^{2}} & -1 \\
-\frac{T_{\mathrm{a}}{ }^{(22)}}{\sqrt{1-\left(T_{\mathrm{a}}{ }^{(32)}\right)^{2}}} & \frac{T_{\mathrm{a}}{ }^{(12)}}{\sqrt{1-\left(T_{\mathrm{a}}{ }^{(32)}\right)^{2}}} & 0 \\
\frac{T_{\mathrm{a}}{ }^{(21)} T_{\mathrm{a}}{ }^{(33)}-T_{\mathrm{a}}{ }^{(31)} T_{\mathrm{a}}{ }^{(23)}}{\left(T_{\mathrm{a}}{ }^{(33)}\right)^{2}+\left(T_{\mathrm{a}}{ }^{(31)}\right)^{2}} & \frac{T_{\mathrm{a}}{ }^{(31)} T_{\mathrm{a}}{ }^{(13)}-T_{\mathrm{a}}{ }^{(11)} T_{\mathrm{a}}{ }^{(33)}}{\left(T_{\mathrm{a}}{ }^{(33)}\right)^{2}+\left(T_{\mathrm{a}}{ }^{(31)}\right)^{2}} & 0
\end{array}\right], \\
& \mathbf{H}_{2}=\mathbf{H}_{3}=\left[\begin{array}{ccc}
\frac{T_{\mathrm{a}}{ }^{(12)} T_{\mathrm{a}}{ }^{(23)}-T_{\mathrm{a}}{ }^{(13)} T_{\mathrm{a}}{ }^{(22)}}{\left(T_{\mathrm{a}}{ }^{(12)}\right)^{2}+\left(T_{\mathrm{a}}{ }^{(22)}\right)^{2}} & 0 & \frac{T_{\mathrm{a}}{ }^{(11)} T_{\mathrm{a}}{ }^{(22)}-T_{\mathrm{a}}{ }^{(12)} T_{\mathrm{a}}{ }^{(21)}}{\left(T_{\mathrm{a}}{ }^{(12)}\right)^{2}+\left(T_{\mathrm{a}}{ }^{(22)}\right)^{2}} \\
\frac{T_{\mathrm{a}}{ }^{(33)}}{\sqrt{1-\left(T_{\mathrm{a}}{ }^{(32)}\right)^{2}}} & 0 & -\frac{T_{\mathrm{a}}{ }^{(31)}}{\sqrt{1-\left(T_{\mathrm{a}}{ }^{(32)}\right)^{2}}} \\
-\frac{T_{\mathrm{a}}{ }^{(31)} T_{\mathrm{a}}{ }^{(32)}}{\left(T_{\mathrm{a}}{ }^{(33)}\right)^{2}+\left(T_{\mathrm{a}}{ }^{(31)}\right)^{2}} & 1 & -\frac{T_{\mathrm{a}}{ }^{(32)} T_{\mathrm{a}}{ }^{(33)}}{\left(T_{\mathrm{a}}{ }^{(33)}\right)^{2}+\left(T_{\mathrm{a}}{ }^{(31)}\right)^{2}}
\end{array}\right] .
\end{aligned}
$$

The UPS for transfer alignment of airborne distributed POS postprocessing can be summarized into two parts: the forward filtering solution and the backward smoothing solution.

Figure 3 shows the data flaw of UPS used in airborne distributed POS and the relation between the state vector estimation of forward filtering $\hat{x}_{k}^{F}$ and backward recursion $\widehat{x}_{k}^{S}$ at time $k$ and $k+1$. In forward filtering solution, the filter results are applied to the nominal trajectory during each filtering step and will be reset to zero after the correction of strapdown navigation (velocity, position, and attitude). The function of the backward smoothing solution is to compensate for the estimation of the forward filter stored during the forward filtering process. The filtering and smoothing are calculated at the main system rate. And after each estimation points, a strapdown inertial navigation algorithm is performed to provide the navigation information.

\section{Simulation and Analysis}

In order to analyze the performance of the proposed UPS, numerical simulation is provided and the comparison with the URTSS using the simulated data of a distributed POS based on one flight trajectory is presented as well.

4.1. Design of Simulation. In this subsection, a typical " $\mathrm{S}+\mathrm{U}$ " shaped flight trajectory of airborne earth observation is designed. Figure 4 shows the plane trajectory and trajectory parameters, respectively. In Figure 4, the AB and CD segment can be considered as the imaging segment. 


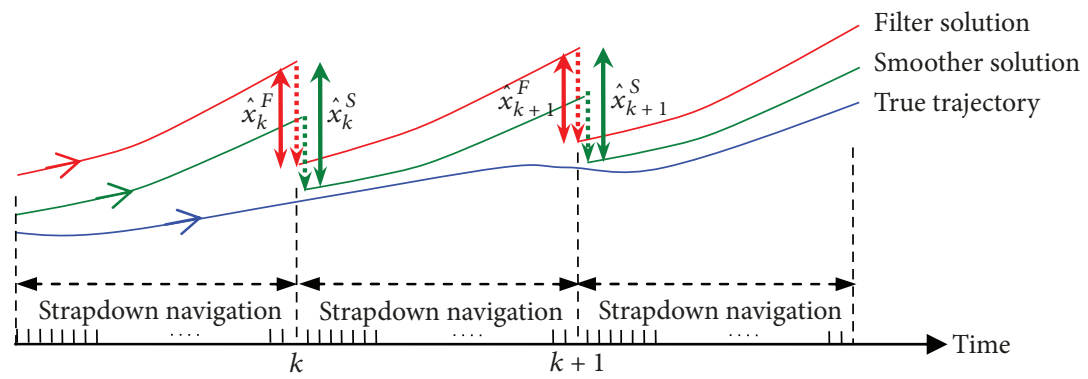

FIgURE 3: The data flaw of UPS used in airborne distributed POS.

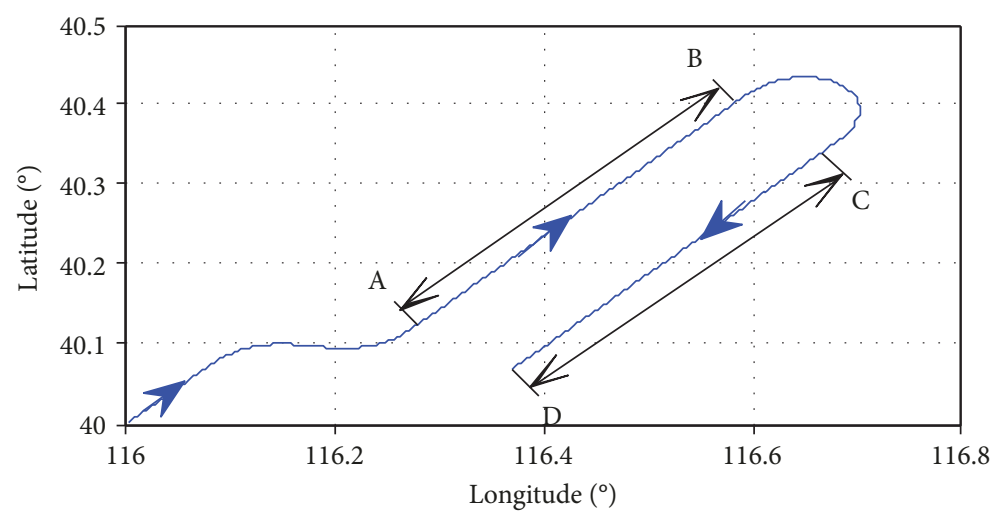

FIGURE 4: Plane trajectory with S-shaped maneuver plus U-shaped flight.

Parameters of flight trajectory are listed as follows: initial latitude is $40^{\circ}$, longitude is $116^{\circ}$, and height is $500 \mathrm{~m}$; initial flight velocity is $100 \mathrm{~m} / \mathrm{s}$; and initial heading angle, pitch angle, and roll angle are $40^{\circ}, 0^{\circ}$, and $0^{\circ}$, respectively. Firstly, the aircraft flies 100 seconds at constant velocity; secondly, the aircraft turns $60^{\circ}$ clockwise (100 seconds) and then turns $60^{\circ}$ anticlockwise (100 seconds); and thirdly, the aircraft flies 400 seconds at constant velocity, finally turns $180^{\circ}$ clockwise (100 seconds), and continually flies 400 seconds at constant velocity. Total flight time is $1300 \mathrm{~s}$.

4.1.1. Data Generation. A trajectory generator is used to generate the theoretical data of the scheduled flight trajectory, which includes position, velocity, attitude, and the output data of gyros and accelerometers. The real outputs of the main system are obtained by adding the corresponding measurement noise to the theoretical position, velocity, and attitude. Then, the theoretical outputs of gyros and accelerometers are converted by rigid misalignment angles and flexure angles, and the constant noise and random noise are added to be the inertial sensor outputs of the subsystem. Meanwhile, the motion parameter benchmarks of subsystem can be obtained through transforming theoretical position, velocity, and attitude by flexure angle.

4.1.2. Parameters of the Main System and Subsystem. The measurement noises of the main system at heading, pitch, roll, and velocity are $0.005^{\circ}(1 \sigma), 0.0025^{\circ}(1 \sigma), 0.0025^{\circ}$ $(1 \sigma)$, and $0.005 \mathrm{~m} / \mathrm{s}(1 \sigma)$, respectively. Both gyro constant drift and random drift of subsystem are $0.01^{\circ} / h$. Both accelerometer constant bias and random bias of the subsystem are $50 \mu g$.

4.1.3. Other Parameters. The rigid misalignment angle of the subsystem relative to the main system is $\rho=$ $\left[\begin{array}{lll}0.5^{\circ} & 0.5^{\circ} & 0.5^{\circ}\end{array}\right]^{\mathrm{T}}$, and the lever arm between the main system and subsystem is $r^{b_{m}}=\left[\begin{array}{lll}5 m & 0.1 m & 0.1 m\end{array}\right]^{\mathrm{T}}$. For multiantenna SAR, these antennae are fixed under both sides of the wing. So the flexure angle rotated around the vertical axis is large, while the flexure angles around the other two axes are small. Accordingly, the correlation time of the second-order Markov processes is selected as $2 \mathrm{~s}, 5 \mathrm{~s}$, and $2 \mathrm{~s}$, and the covariances of flexure angles are $0.01,0.15$, and 0.01 , respectively. The curves of flexure angle and flexure angle rate selected in this simulation are shown in Figures 5 and 6 . All the initial attitude errors are $40^{\circ}$. The particle number is 100 . The data update rate of the subsystem is $100 \mathrm{~Hz}$ and the filter frequency is $1 \mathrm{~Hz}$.

4.2. Simulation Results and Analysis. The differences between theoretical motion parameters of subsystem and their estimates obtained from estimation, called estimate errors, are used to assess and compare the performance of UPS and URTSS.

Figures 7-10 show the estimate error curves of UPS and URTSS, including the estimate errors of attitude, velocity, position, and baseline. The means of root mean square error (RMSE) and standard deviation (STD) values of motion information estimate errors in imaging segments $\mathrm{AB}$ and 

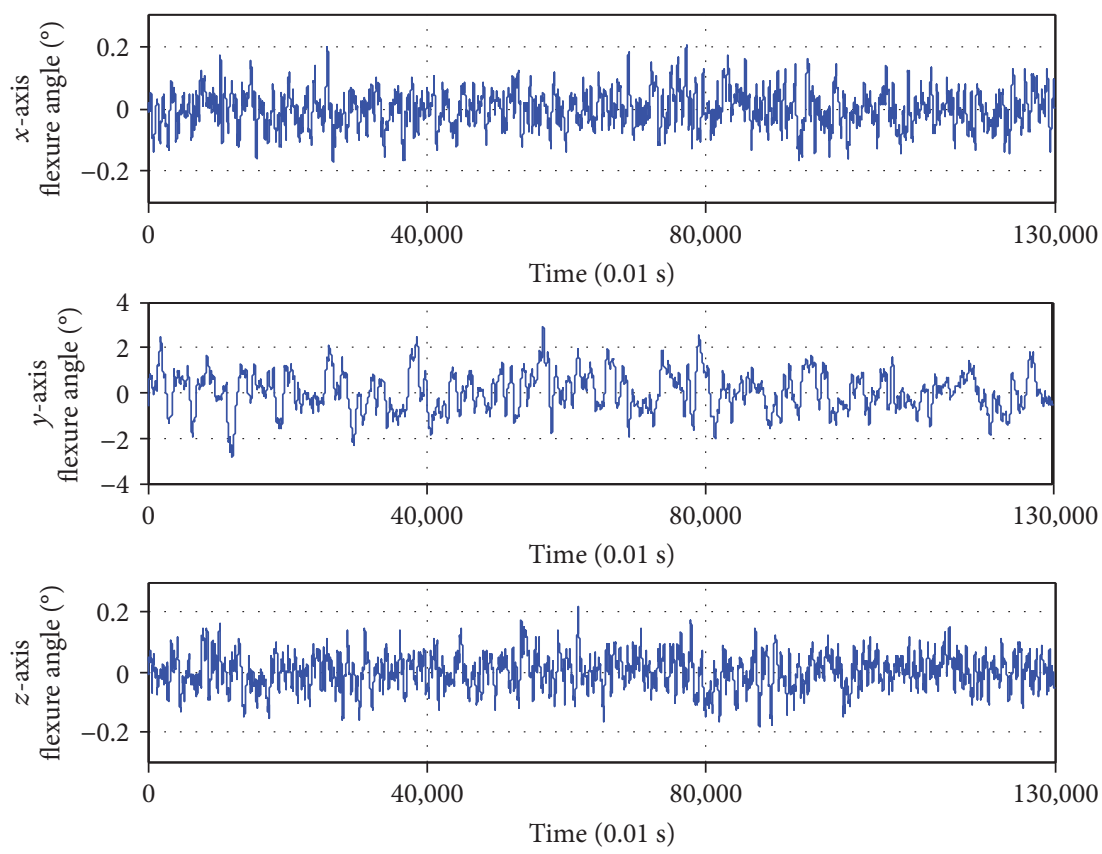

Figure 5: Flexure angle on $x$-axis, $y$-axis, and $z$-axis.
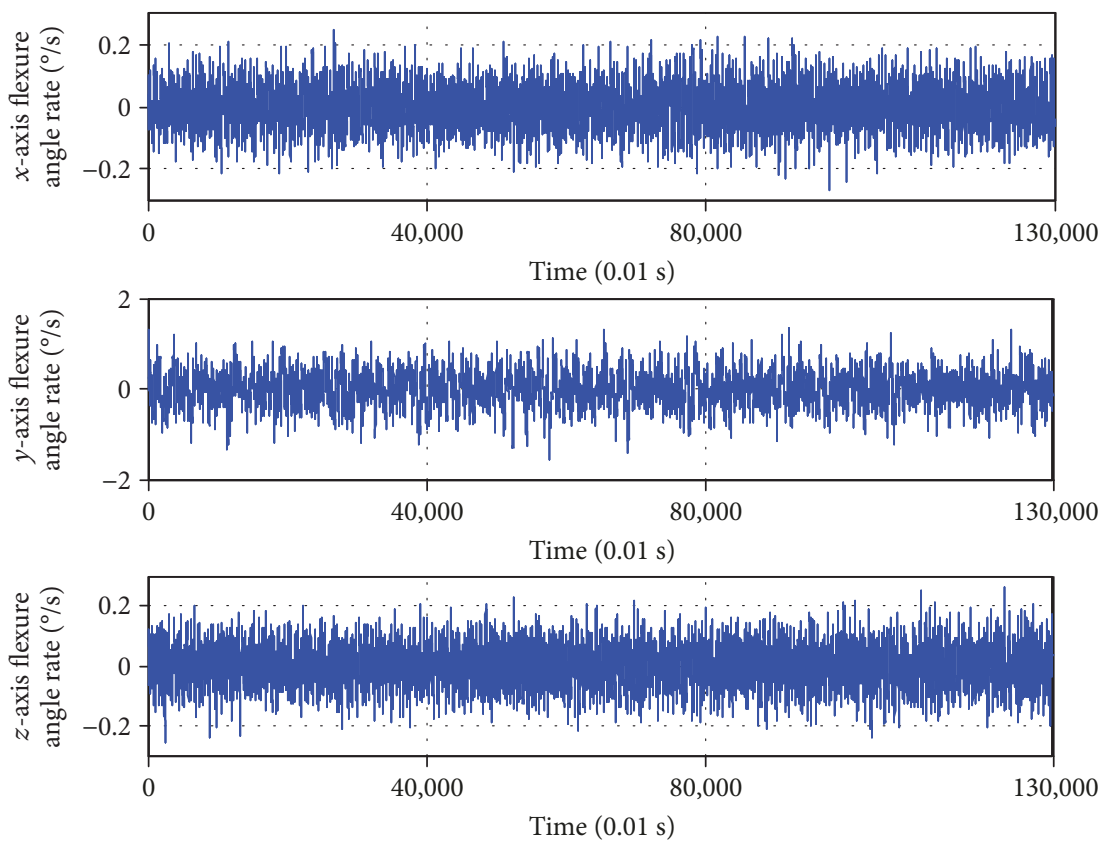

Figure 6: Flexure angle rate on $x$-axis, $y$-axis, and $z$-axis.

$\mathrm{CD}$ are calculated and shown in Table 1. The calculation formulas of RMSE and STD are given as follows:

$$
\begin{aligned}
\text { RMSE } & =\sqrt{\frac{\sum_{i=1}^{n}\left(e_{i}\right)^{2}}{n^{\prime}}}, \\
\text { STD } & =\sqrt{\frac{\sum_{i=1}^{h}\left(e_{i}-\bar{e}\right)^{2}}{n^{\prime}}},
\end{aligned}
$$

where $e_{i}$ denotes the $i$ th estimate error, $n^{\prime}$ denotes the number of estimate error, and $\bar{e}$ denotes the mean of all estimate error.

For attitude estimation, from Figure 7 and Table 1, we can see that there is a large and obvious trend item in the heading estimation error of URTSS, and the estimation error of URTSS on pitch and roll is prone to be affected by maneuver and their value are bigger than UPS even after $180^{\circ}$ clockwise. Compared with URTSS, when the 

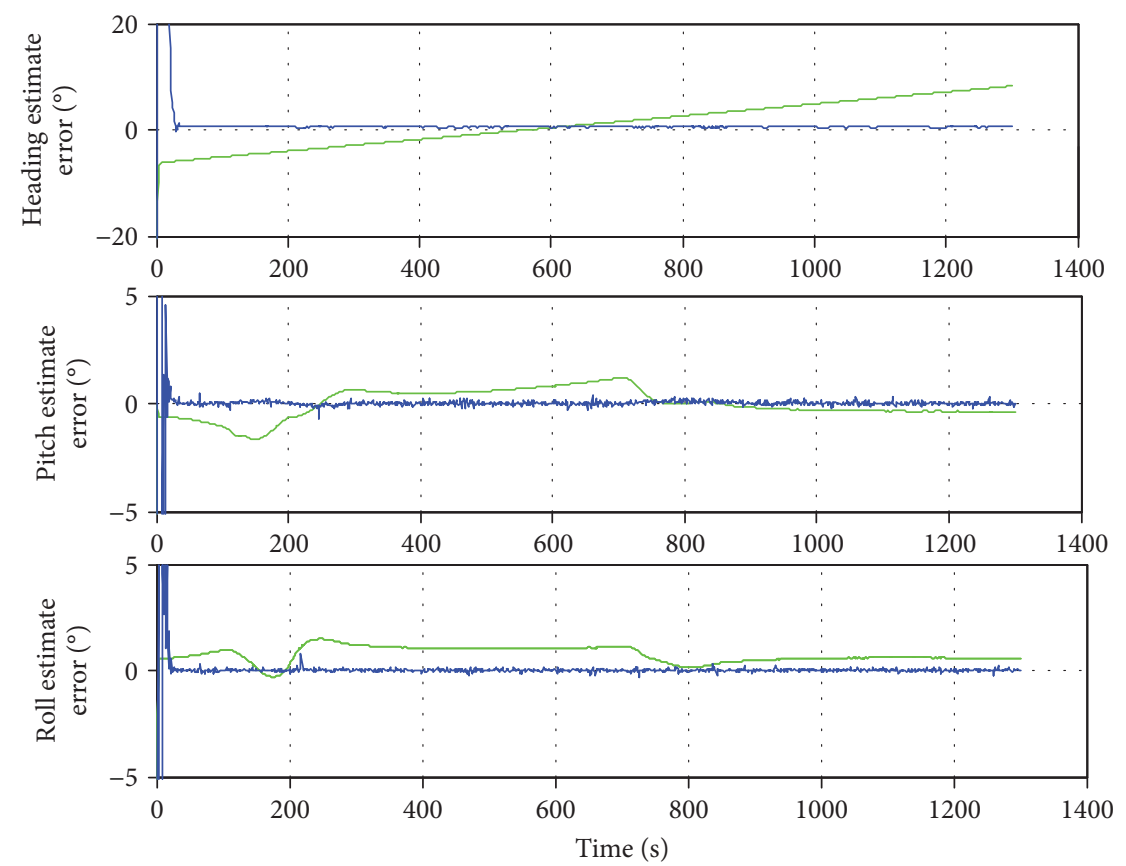

- URTSS

- UPS

FIGURE 7: Attitude estimate errors of UPS and URTSS.
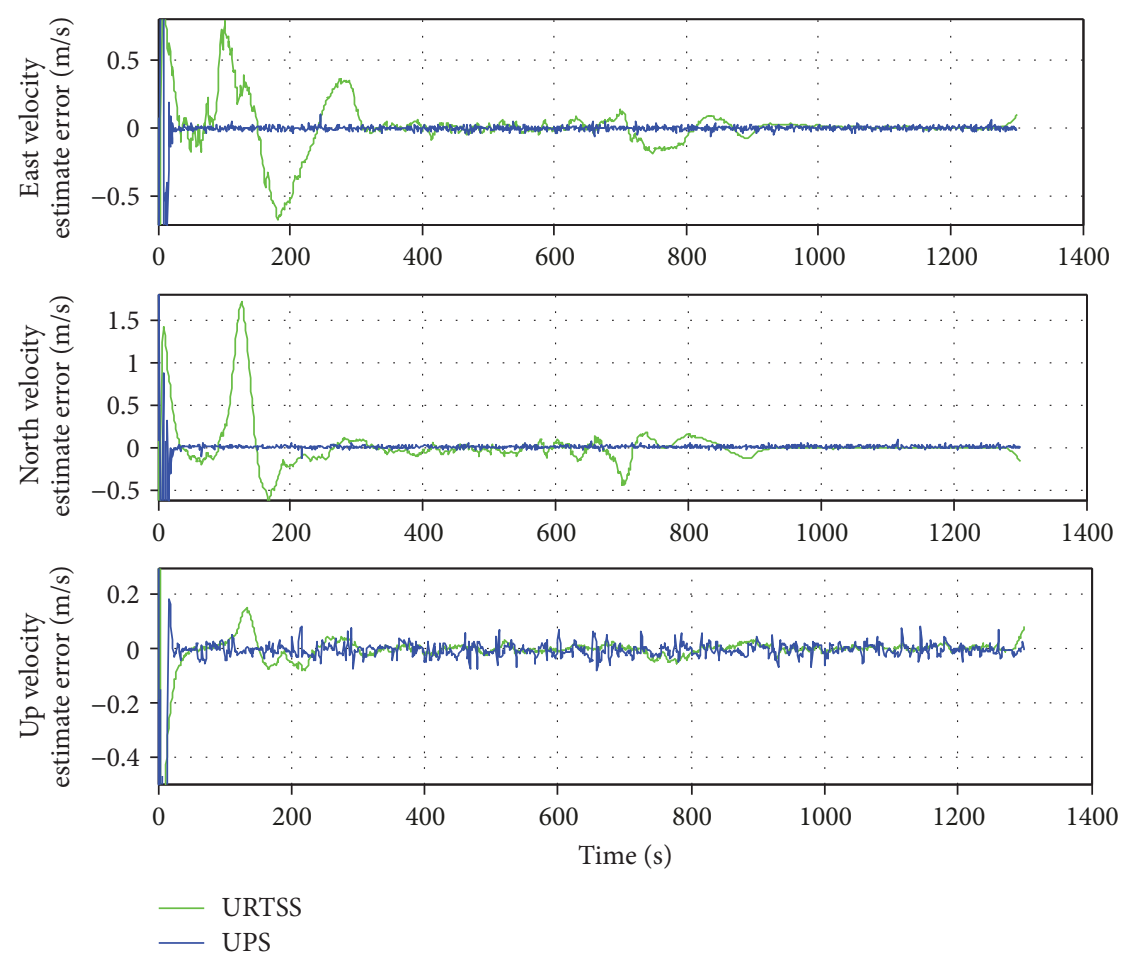

FIGURE 8: Velocity estimate errors of UPS and URTSS.

initial misalignment angle is $40^{\circ}$, the attitude estimation error of UPS is significantly reduced and the error curves are more stable both on $\mathrm{AB}$ and $\mathrm{CD}$ segments; the estimation accuracy on heading and pitch of UPS is obviously better than that of URTSS, and only the STD on roll of UPS is slightly lower than that of URTSS. 

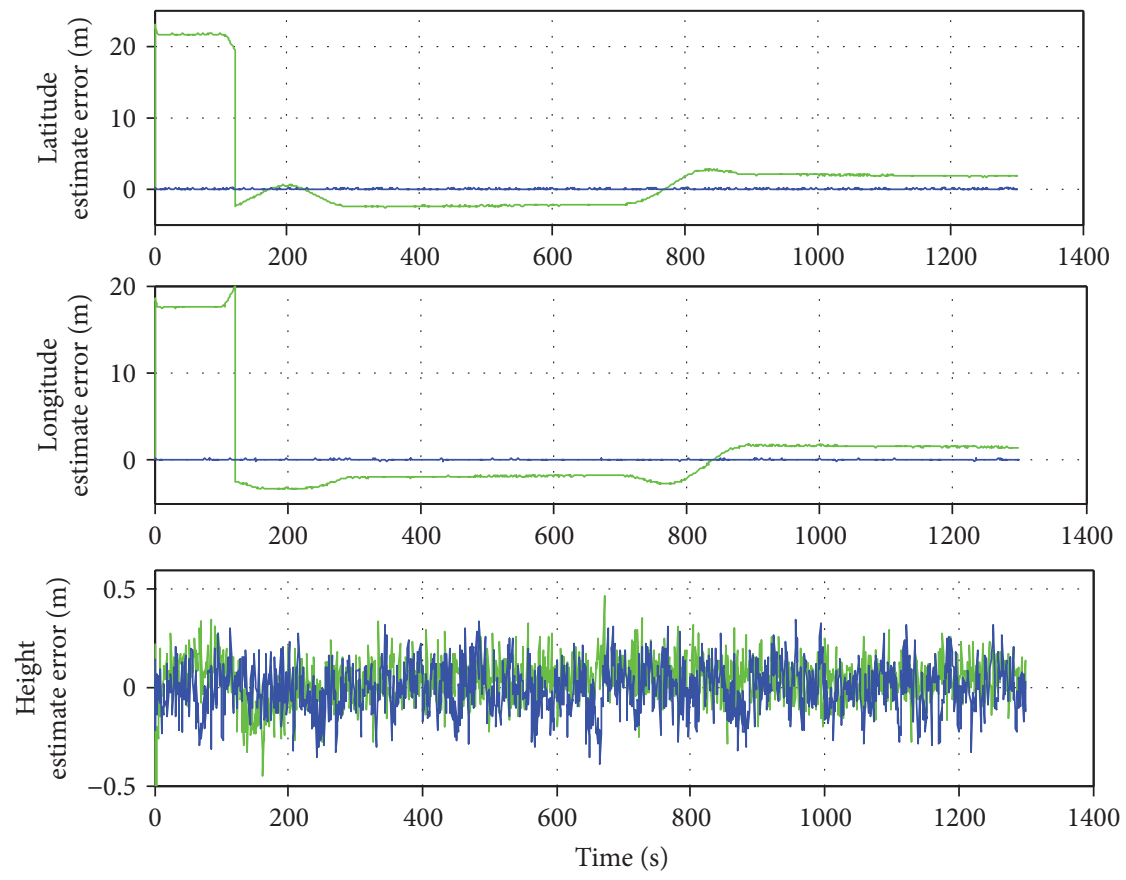

— URTSS

- UPS

FIGURE 9: Position estimate errors of UPS and URTSS.

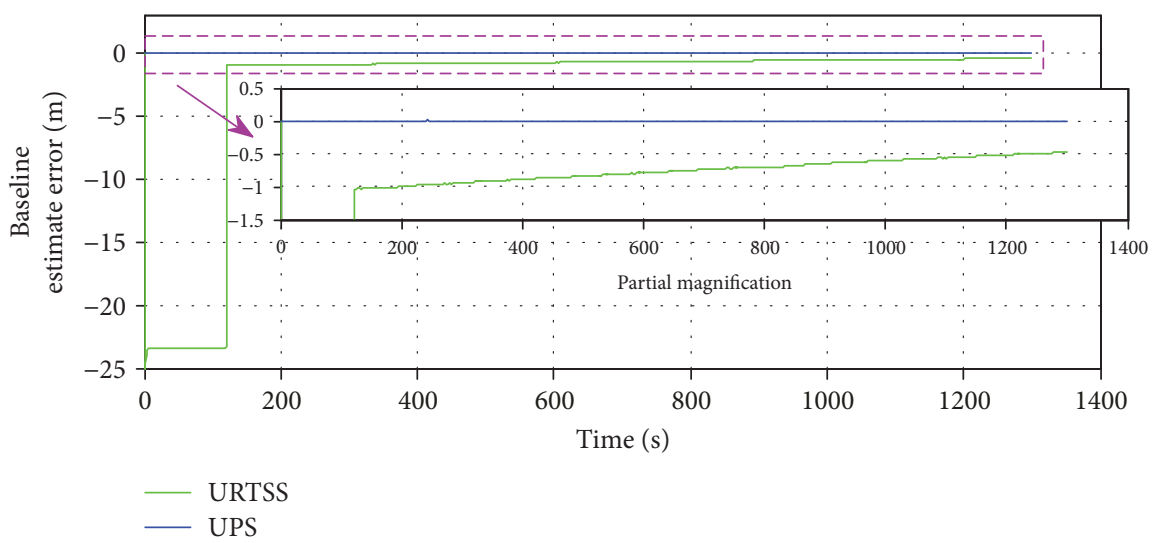

FIGURE 10: Baseline estimate errors of UPS and URTSS.

For velocity estimation, Figure 8 and Table 1 show that the estimation accuracy on east velocity and north velocity of UPS is better than that of URTSS and the error curves of URTSS are prone to be affected by maneuver, especially in the initial ' $S$ ' shape flight, the estimation error of velocity of URTSS is unstable and very large; the estimation accuracy on up velocity of UPS is lower than that of URTSS.

For position estimation, Figure 9 and Table 1 show that the estimation accuracy on the latitude and longitude of UPS is better than that of URTSS, and the estimation accuracy on the height of UPS is lower than that of URTSS, which corresponds to the up velocity error result. The estimation error of latitude and longitude of URTSS in the initial 'S' shape flight (from $0 \mathrm{~s}$ to $300 \mathrm{~s}$ ) is very large due to the large velocity error in this stage.

Besides, the space distance between the main system and subsystem (i.e., the baseline between two antennas of SAR) is a very important parameter for interference precision of interferometric SAR and the higher the measurement accuracy of the baseline, the higher the object's digital elevation model accuracy of the interferometric SAR. Here, the baseline error is calculated by theoretical position and real position output of the main system and subsystem. From Figure 10 and Table 1, we can see that the baseline error curve of UPS is more stable and the baseline estimation accuracy of UPS is better than that of URTSS both on RMSE and STD. 
TABLE 1: Means of RMSE and STD values of estimate errors.

\begin{tabular}{lccccc}
\hline \multirow{2}{*}{ Parameter } & & \multicolumn{2}{c}{ URTSS } & \multicolumn{2}{c}{ UPS } \\
& & RMSE & STD & RMSE & STD \\
\hline \multirow{4}{*}{ Attitude error } & Heading $\left(^{\circ}\right)$ & 3.7874 & 1.2914 & 0.4959 & 0.0217 \\
& Pitch $\left({ }^{\circ}\right)$ & 0.5166 & 0.1218 & 0.0766 & 0.0781 \\
& Roll $\left(^{\circ}\right)$ & 0.8238 & 0.0371 & 0.0569 & 0.0568 \\
\hline \multirow{3}{*}{ Velocity error } & East $(\mathrm{m} / \mathrm{s})$ & 0.0264 & 0.0245 & 0.0133 & 0.0134 \\
& North $(\mathrm{m} / \mathrm{s})$ & 0.0403 & 0.0414 & 0.0225 & 0.0148 \\
& Up $(\mathrm{m} / \mathrm{s})$ & 0.0125 & 0.0124 & 0.0224 & 0.0211 \\
\hline \multirow{3}{*}{ Position error } & Latitude $(\mathrm{m})$ & 2.1444 & 0.0990 & 1.0077 & 0.0529 \\
& Lengitude $(\mathrm{m})$ & 1.7280 & 0.0878 & 0.0510 & 0.0495 \\
\hline Baseline error & $(\mathrm{m})$ & 0.7039 & 0.0532 & 0.0014 & 0.0008 \\
\hline
\end{tabular}

From the simulation result, we can see that the estimation accuracy of motion parameters of the proposed method is higher than that of the URTSS as a whole.

\section{Conclusion}

In this paper, a nonlinear smoother called UPS has been proposed to deal with the off-line transfer alignment estimation of airborne distributed POS. In the UPS, UKF, PF, and smoothing are combined together to provide the respective advantages from each of them to improve estimation accuracy. The validity of the proposed algorithm is verified by simulation test and comparison with URTSS on the estimation accuracy of motion parameters and baseline. The simulation results show that UPS can improve estimation accuracy and has more adaptability to the maneuver than that of URTSS. This method is a better choice for off-line transfer alignment in distributed POS with large misalignment and will greatly improve the resolution of flexible baseline interferometric SAR imaging. The next work is that the practical flight experiment based on long flexible baseline will be implemented to validate the performance of this method.

\section{Data Availability}

The data used to support the findings of this study are available from the corresponding author upon request.

\section{Conflicts of Interest}

The authors declare that they have no conflicts of interest.

\section{Acknowledgments}

This work was supported in part by the National Natural Science Foundation of China (Grant nos. 61473020 and 61721091), International (Regional) Cooperation and Communication Project (Grant no. 61661136007), and Fundamental Research Funds for the Central Universities.

\section{References}

[1] J. Yang, W. Su, and H. Gu, "High-resolution multiple-inputmultiple-output-inverse synthetic aperture radar imaging based on sparse representation," IET Radar, Sonar \& Navigation, vol. 10, no. 7, pp. 1277-1285, 2016.

[2] L. Gao, Y. Zeng, and L. Wang, "Design of Array for Airborne Forward Looking SAR Based on Compressed Sensing," in 2014 IEEE International Conference on Signal Processing, Communications and Computing (ICSPCC), pp. 473-476, Guilin, China, August 2014.

[3] L. T. Zeng, Y. Huai, Z. Y. Li, M. D. Xing, and Y. Liang, "Two-dimensional autofocus technique for high-resolution spotlight synthetic aperture radar," IET Signal Processing, vol. 10, no. 6, pp. 699-707, 2016.

[4] D. Li, M. Zhong, and D. Guo, "Error Detection and Compensation in Transfer Alignment for the Distributed POS," in 2013 25th Chinese Control and Decision Conference (CCDC), pp. 4194-4199, Guiyang, China, May 2013.

[5] X. Gong and J. Zhang, "An Innovative Transfer Alignment Method Based on Federated Filter for Airborne Distributed POS,” Measurement, vol. 86, pp. 165-181, 2016.

[6] L. Song, Z. Duan, and J. Sun, "Application of Ho filter on the angular rate matching in the transfer alignment," Discrete Dynamics in Nature and Society, vol. 2016, Article ID 4859475, 9 pages, 2016.

[7] J. Sun, X. S. Xu, Y. T. Liu, T. Zhang, and Y. Li, "Initial alignment of large azimuth misalignment angles in SINS based on adaptive UPF," Sensors, vol. 15, no. 9, pp. 21807-21823, 2015.

[8] D. Wang, H. Lv, and J. Wu, "In-flight Initial Alignment for Small UAV MEMS-based Navigation Via Adaptive Unscented Kalman Filtering Approach," Aerospace Science and Technology, vol. 61, pp. 73-84, 2017.

[9] J. L. Crassidis, "Sigma-point Kalman filtering for integrated GPS and inertial navigation," IEEE Transactions on Aerospace and Electronic Systems, vol. 42, no. 2, pp. 750-756, 2006.

[10] G. Chang and M. Liu, "Hybrid Kalman and Unscented Kalman filters for INS/GPS integrated system considering constant lever arm effect," Journal of Central South University, vol. 22, no. 2, pp. 575-583, 2015.

[11] A. Farina, B. Ristic, and D. Benvenuti, "Tracking a Ballistic Target: Comparison of Several Nonlinear Filters," IEEE Transactions on Aerospace and Electronic Systems, vol. 38, no. 3, pp. 854-867, 2002.

[12] H. Liu, L. Yu, C. Ruan, and Z. Zhou, “Tracking air-to-air Missile Using Proportional Navigation Model with Genetic Algorithm Particle Filter," Mathematical Problems in Engineering, vol. 2016, Article ID 3921608, 11 pages, 2016.

[13] I. Moir, A. G. Seabridge, and M. Jukes, Military Avionics Systems, Thomson Press, New Delhi, India, 2005.

[14] N. J. Gordon, D. J. Salmond, and A. F. M. Smith, "Novel approach to nonlinear/non-Gaussian Bayesian state estimation," IEE Proceedings F Radar and Signal Processing, vol. 140, no. 2, p. 107, 1993.

[15] R. V. D. Merwe, A. Doucet, N. D. Freitas, and E. Wan, "The Unscented Particle Filter," in Proceedings of the 13th International Conference on Neural Information Processing Systems, pp. 563-569, Hong Kong, China, 2006.

[16] J. S. Meditch, "A survey of data smoothing for linear and nonlinear dynamic systems," Automatica, vol. 9, no. 2, pp. 151-162, 1973. 
[17] S. Särkkä, "Unscented Rauch-Tung-Striebel Smoother," IEEE Transactions on Automatic Control, vol. 53, no. 3, pp. 845-849, 2008.

[18] X. Gong, R. Zhang, and J. Fang, “Application of unscented R-T-S smoothing on INS/GPS integration system post processing for airborne earth observation," Measurement, vol. 46, no. 3, pp. 1074-1083, 2013.

[19] G. Kitagawa, "Monte Carlo filter and smoother for nonGaussian nonlinear state space models," Journal of Computational and Graphical Statistics, vol. 5, no. 1, pp. 1-25, 1996.

[20] J. Frydendall, "Particle Filters with Applications," Technical Report, University of Denmark, DTU Informatics, No. 200902, 2009.

[21] D. Jeon and Y. Eun, "Distributed asynchronous multiple sensor fusion with nonlinear multiple models," Aerospace Science and Technology, vol. 39, pp. 692-704, 2014.

[22] Y. Meng, S. Gao, and W. Wang, "Unscented Particle Filter Based Gaussian Process Regression for IMU/BDS Train Integrated Positioning," in 2016 IEEE Information Technology, Networking, Electronic and Automation Control Conference, pp. 1070-1073, Chongqing, China, May 2016.

[23] J. Cheng, T. Wang, D. Guan, and M. Li, "Polar Transfer Alignment of Shipborne SINS with a Large Misalignment Angle," Measurement Science \& Technology, vol. 27, no. 3, article 035101, 2016.

[24] D. Goshen-Meskin and I. Y. Bar-itzhack, "Unified approach to inertial navigation system error modeling," Journal of Guidance, Control and Dynamics, vol. 15, no. 3, pp. 648-653, 1992.

[25] Y. Lu and X. Cheng, "Random misalignment and lever arm vector online estimation in shipborne aircraft transfer alignment," Measurement, vol. 47, no. 1, pp. 756-764, 2014. 


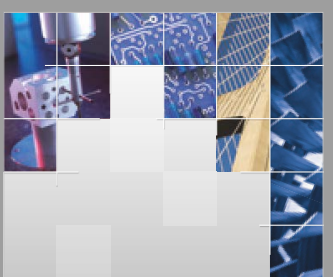

\section{Enfincering}
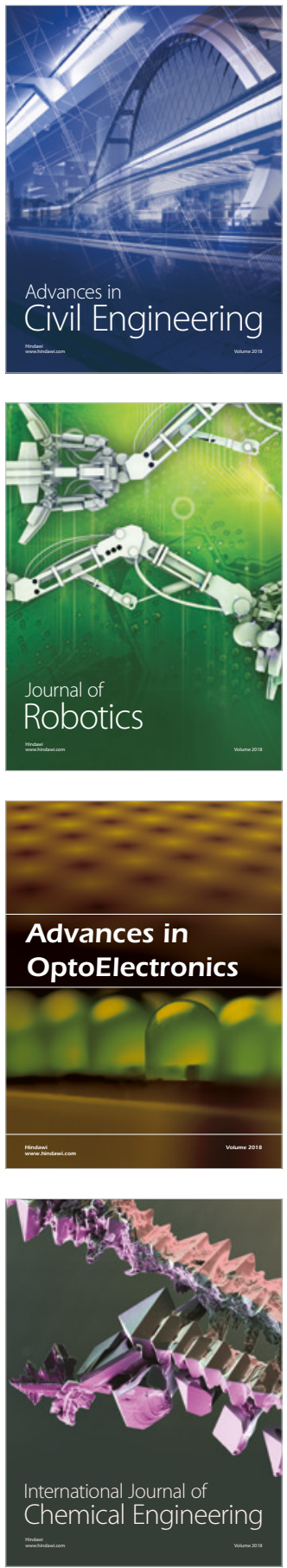

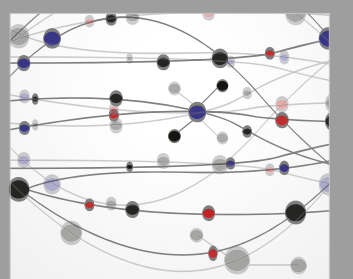

\section{Rotating \\ Machinery}

The Scientific World Journal

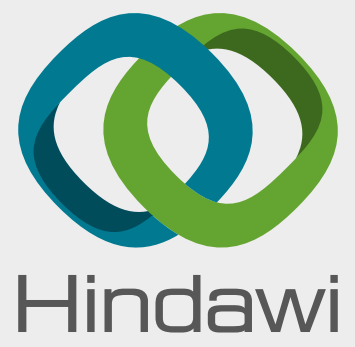

Submit your manuscripts at

www.hindawi.com
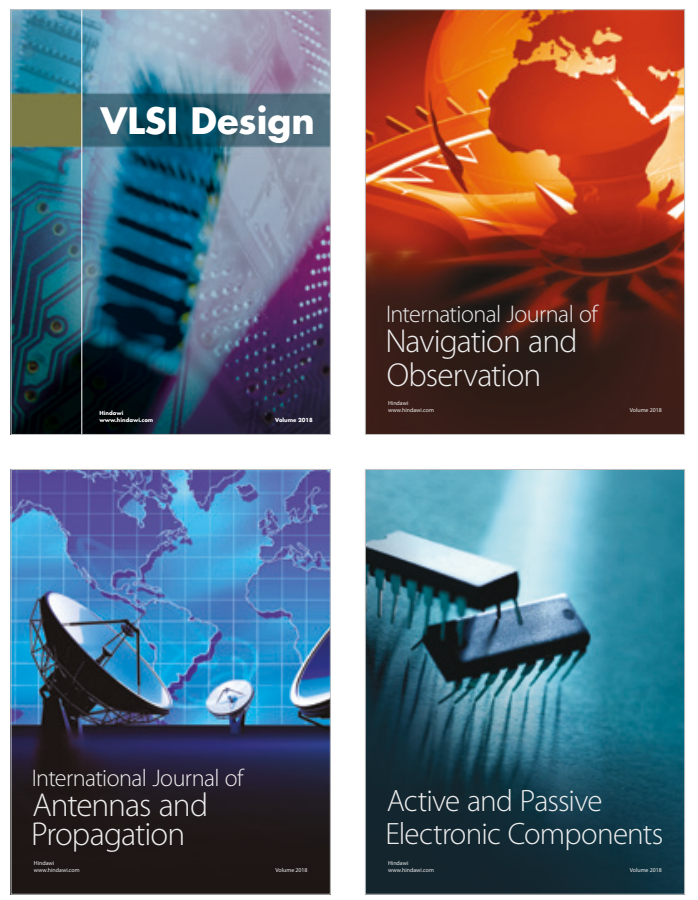
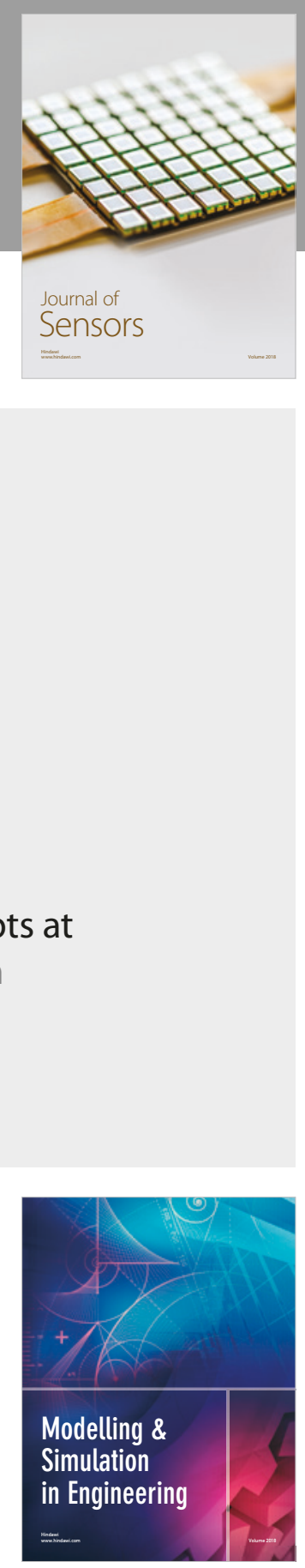

\section{Advances \\ Multimedia}
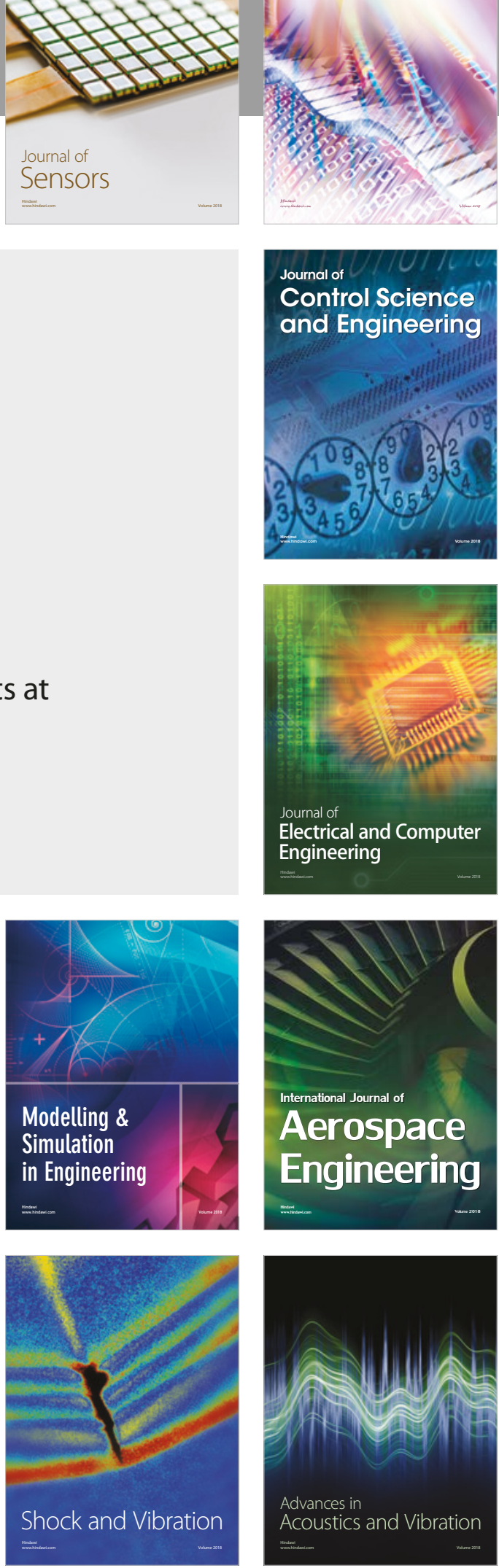